\title{
The PACINPAT study: physical activity counselling in in-patients with major depressive disorders
}

\author{
DEPRESSION / EXERCISE IS MEDICINE / MONITORING / PA PROMOTION / TRAINING
}

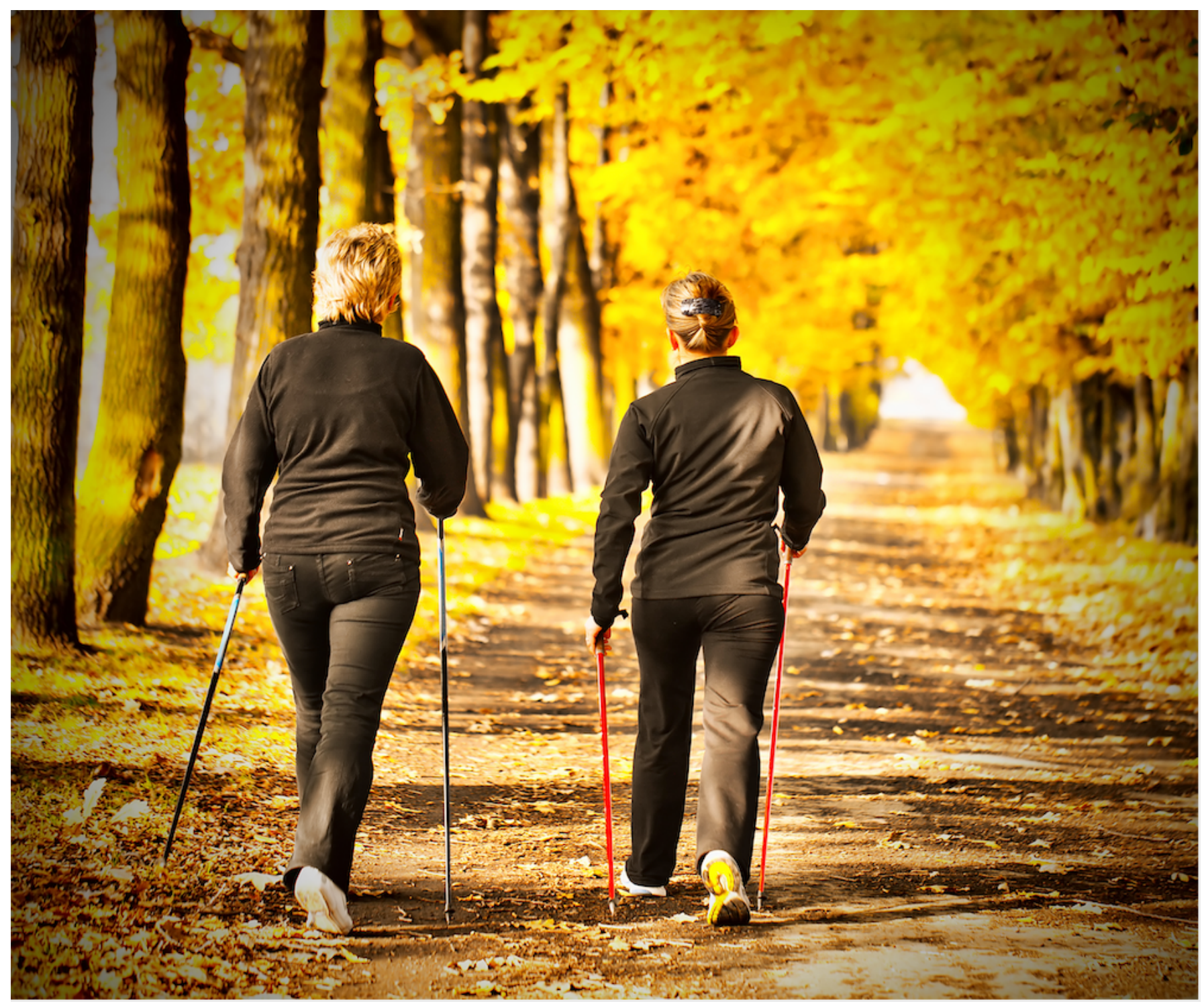

Kreppke Jan-Niklas ${ }^{1}$, Cody Robyn ${ }^{2}$, Imboden Christian ${ }^{3}$, Faude Oliver ${ }^{1}$, Gerber Markus ${ }^{2 *}$

${ }^{1}$ Exercise and Movement Science, Department of Sport, Exercise and Health, University of Basel, Switzerland 
${ }^{2}$ Sport Science and Psychosocial Health, Department of Sport, Exercise and Health, University of Basel, Switzerland

${ }^{3}$ Private Clinic Wyss AG, Münchenbuchsee, Switzerland

* on behalf of the PACINPAT study group

\section{Introduction}

Major depressive Disorder (MDD) is a widespread and burdensome disease. People with MDD suffer from loss of interest and pleasure in activities that they would usually enjoy. In addition, they report anxiety, complex somatic pain syndromes, cognitive restrictions, loss of sexual interest, impaired sleep and social withdrawal. MDD is the leading cause for years lived with disability (YLD) in women and men and has a lifetime prevalence of $10-20 \%$.

Standard treatment for MDD includes pharmacological, non-pharmacological or combinations of both. Pharmacological treatment is the most established, however, due to poor adherence and clinically relevant negative side effects such as sexual dysfunction and weight gain, acceptability in some patients is low. Additionally, treatment resistance emerges in approximately $30 \%$ of cases.

Consequently, non-pharmacological therapies should be increasingly promoted as an add-on to standard treatment to support the recovery and minimize the risk of relapse. Physical activity (PA) promotion is an effective method of treatment. Beyond antidepressant effects, beneficial impacts of PA are seen in physical (improvement of cardiorespiratory fitness) and psychological (distraction from negative thoughts) outcomes. Research has shown a moderate to large effect of endurance exercise and a large effect of neuromuscular exercise compared to control groups on reducing depressive symptoms [1]. Despite the positive effects of PA, people with MDD find it particularly difficult to engage in regular PA due to depressive symptoms such as lethargy or low levels of motivation [2].

In order to increase PA, effective programs targeting behavioral change are necessary [3]. In healthy but inactive participants, the use of individual telephone coaching and the delivery of behavioral change techniques (BCTs) has proven effective in supporting the initiation of PA. In a randomized controlled trial (RCT), PA increased in the intervention group compared to the placebo control group after a six months follow-up [4].

Given this knowledge, it is important to adapt existing programs to support the increase of PA for patients with MDD [5]. Therefore, we planned the PACINPAT study, in which lifestyle PA coaching for inactive people suffering from MDD is integrated in a clinical setting. The aim of the study is to increase the knowledge of PA levels in this population and to evaluate the efficacy of the coaching program.

\section{Methods}

The PACINPAT study is a multi-center RCT including an intervention, placebo and healthy control group [6]. Participants are recruited and assessed in four Swiss psychiatric clinics: Clinic Wyss Münchenbuchsee, University Psychiatric Hospital Basel, Clinic Sonnenhalde Riehen and Psychiatric Services Solothurn. Women and men are eligible to be included in the study if they are between 18 and 65 years, physically inactive (< 150 minutes of moderate-to-vigorous physical activity per week), suffering from MDD according 
to ICD-10 and command of sufficient German language skills. Overall, 334 participants will be recruited and randomly assigned to the intervention or the placebo control group. Data assessments take place during the hospitalization (baseline), six weeks and one year after leaving the clinic. The intervention is a lifestyle PA coaching partly based on the MoVo-intervention featuring the strengthening of motivational and volitional processes and the use of appropriate BCTs selected form the Behavior Change Wheel [7]. The intervention group receives two face-to-face coaching sessions by their personal PA coach during the inpatient treatment, followed by bi-weekly telephone calls, which are supported by the use of a mutually accessible smartphone-app. Action planning, goal setting, barrier management, changing social and physical environment as well as social support are main contents. The app facilitates planning and selfmonitoring. The intervention lasts one year, consisting of 26 coaching sessions and is performed by trained PA coaches. The control group also receive two face-to-face meetings with the PA coach during which they receive generic recommendations regarding health enhancing PA. The healthy control group ( $\mathrm{n}=167)$ is recruited from the general population with the same eligibility criteria, except for the MDD diagnosis. This group acts as an age and gender matched comparison at baseline. The main outcome of interest is objectively measured PA one year after discharge from inpatient treatment. It is measured via hip worn accelerometer. Secondary outcomes are self-reported PA, cardiovascular risk markers, psychological determinants of PA and depression severity. Additionally, a nested qualitative study will take place to elicit the lived experience of the participants in the intervention group. First results comparing in-patients and the healthy control group are expected in early 2021. Intervention results will be available in late 2022.

\section{Conclusion}

This is the first study implementing PA coaching for people suffering from MDD. If an increase in PA levels leads to physiological, psychological and social benefits as expected, an improvement of health and quality of life is given. In addition, this study will provide valuable insights into the feasibility of implementing such an intervention in psychiatric clinics systematically, thus further enriching in-patient care.

\section{Corresponding author}

Prof. Dr. Markus Gerber

Sport Science and Psychosocial Health

Department of Sport, Exercise and Health

University of Basel, Switzerland

markus.gerber@unibas.ch

\section{References}

1. Nebiker L, Lichtenstein E, Minghetti A, Zahner L, Gerber M, Faude O, et al. Moderating effects of exercise duration and intensity in neuromuscular vs. endurance exercise interventions for the treatment of depression: a meta-analytical review. Frontiers in psychiatry. 2018;9:305.

2. Gerber M, Holsboer-Trachsler E, Pühse U, Brand S. Exercise is medicine for patients with major depressive disorders: but only if the "pill" is taken! Neuropsychiatric disease and treatment. 2016;12:1977.

3. Gerber M, Fuchs R, Pühse U. Follow-up of a short motivational and volitional exercise-intervention 
trial with overweight and obese individuals. Schweizerische Zeitschrift fur Sportmedizin und Sporttraumatologie. 2010;58(4):108.

4. Fischer X, Kreppke J-N, Zahner L, Gerber M, Faude O, Donath L. Telephone-Based Coaching and Prompting for Physical Activity: Short-and Long-Term Findings of a Randomized Controlled Trial (Movingcall). International Journal of Environmental Research and Public Health. 2019;16(14):2626.

5. Brand S, Colledge F, Beeler N, Pühse U, Kalak N, Bahmani DS, et al. The current state of physical activity and exercise programs in German-speaking, Swiss psychiatric hospitals: results from a brief online survey. Neuropsychiatric disease and treatment. 2016;12:1309.

6. Gerber M, Beck J, Brand S, Cody R, Donath L, Eckert A, et al. The impact of lifestyle Physical Activity Counselling in IN-PATients with major depressive disorders on physical activity, cardiorespiratory fitness, depression, and cardiovascular health risk markers: study protocol for a randomized controlled trial. Trials. 2019;20(1):367.

7. Michie S, Van Stralen MM, West R. The behaviour change wheel: a new method for characterising and designing behaviour change interventions. Implementation science. 2011;6(1):42. 